\title{
Initiating a research-focused academic career in chemical engineering: Perspectives from faculty at different career stages
}

\author{
Viktor J. Cybulskis $^{1}$ | Rajamani Gounder ${ }^{2}$ | Sarah Mojarad ${ }^{3}$ | Mark E. Davis ${ }^{4}$ \\ ${ }^{1}$ Department of Biomedical and Chemical Engineering, Syracuse University, Syracuse, New York \\ ${ }^{2}$ Charles D. Davidson School of Chemical Engineering, Purdue University, West Lafayette, Indiana \\ ${ }^{3}$ Viterbi School of Engineering and Keck School of Medicine, University of Southern California, Los Angeles, California \\ ${ }^{4}$ Chemical Engineering, California Institute of Technology, Pasadena, California \\ Correspondence \\ Mark E. Davis, Chemical Engineering, California Institute of Technology, Pasadena, CA 91125. \\ Email: mdavis@cheme.caltech.edu
}

KEYWORDS: assistant professor, faculty candidate, job search, research program tenure-track

\section{1 | INTRODUCTION}

Each fall, eager young researchers participate in the Meet the Faculty Candidates poster session at the AIChE Annual Meeting, and many more apply to tenure-track faculty openings at academic institutions across the United States and throughout the world. These individuals embark on this journey with the eventual goal of becoming full professors. The process of initiating an academic career and developing a successful independent research program is an arduous journey that involves multiple stages. These stages include being hired into a faculty position, building an independent research program that involves recruiting students and can include constructing a laboratory, identifying research areas and specific problems to investigate, and establishing oneself as a scientific leader of a particular subject matter area within a broader community. Faculty candidates commonly seek advice from mentors or peers who have recently navigated the faculty interview and hiring processes to successfully obtain an academic position. Additionally, they often review the wealth of resources that are available on the Internet and in print. However, it can be daunting to sieve through this collective knowledge base to identify relevant information, as it invariably contains conflicting viewpoints and advice that may be subjective, generic to any research-focused faculty position, or highly field specific.
Here, we provide a perspective that is a high-level overview of the general hiring process for tenure-track chemical engineering faculty in research-focused departments in the United States. We offer guidance based on our personal experiences, anecdotes, and observations that we have found to be most relevant and valuable. For the purpose of discussion, we have broken down the process into four phases with the approximate timeframes, as illustrated in Figure 1, to address key aspects of preparing for a faculty career. These phases involve the processes of preparing during your training as a graduate student and postdoctoral researcher, applying to open faculty positions, interviewing and negotiating for a position, and establishing an impactful research program. This insight has been collected, curated, and passed down over the past three decades in the Mark E. Davis laboratory at the California Institute of Technology that has mentored and produced nearly 40 faculty members during this time period. As with all advice, the contents of this article are intended to be neither prescriptive nor comprehensive, but rather to provoke thought and to share suggestions that have been helpful for a large number of future faculty members who trained in the Davis laboratory. Based on the success of these individuals in navigating the hiring process to obtain faculty positions, along with the advice they have subsequently passed on to their students, numerous people have asked us to share this information. We hope that this perspective serves that purpose.

This is an open access article under the terms of the Creative Commons Attribution License, which permits use, distribution and reproduction in any medium, provided the original work is properly cited.

(C) 2020 The Authors. AIChE Journal published by Wiley Periodicals, Inc. on behalf of American Institute of Chemical Engineers. 


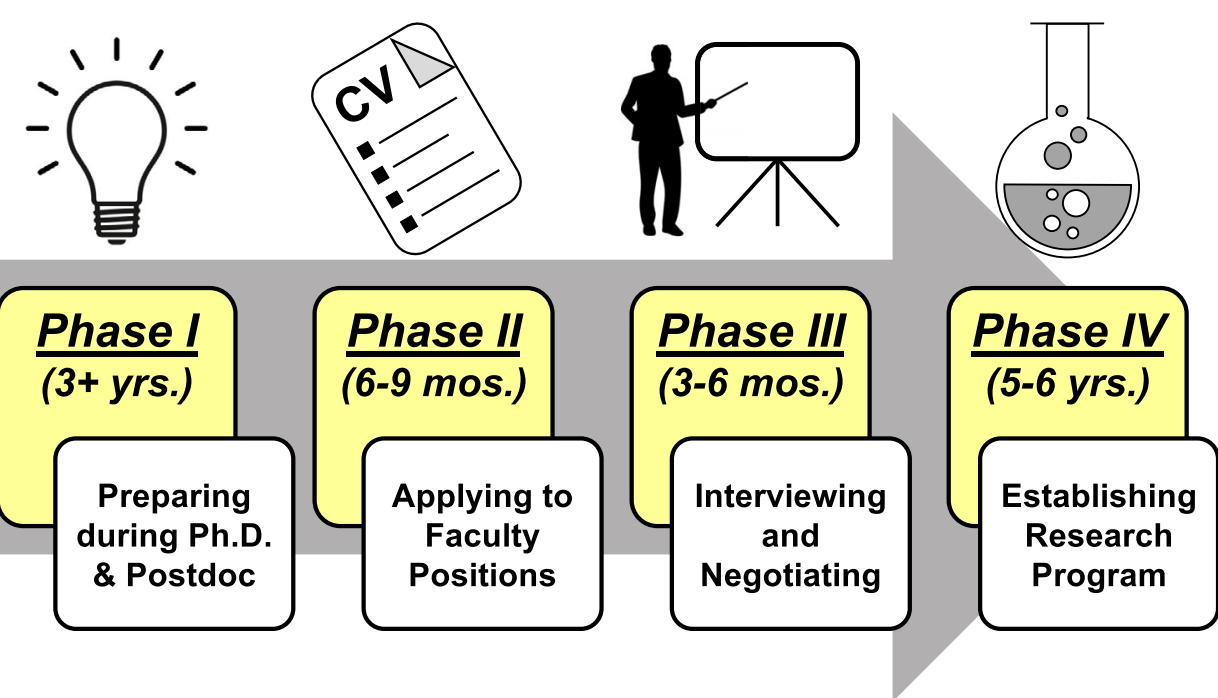

FIGURE 1 Approximate phases and timelines for initiating a researchfocused faculty career [Color figure can be viewed at wileyonlinelibrary.com]

\section{2 | PHASE I: PREPARING DURING GRADUATE AND POSTDOCTORAL STUDY (3+ YEARS)}

\section{1 | Developing your skill set}

Early in graduate school, it is important to pursue electives that provide you with a broader foundational background in your particular area of study. While your Ph.D. research should focus on the fundamental aspects of a problem to advance your field beyond the current state-of-the-art, you should also take advantage of opportunities to enhance your breadth of knowledge, to better prepare you to address a broader range of future research topics. This is the time to gain a deep understanding of the science and engineering necessary to successfully launch your academic career, because the further you proceed in your profession, the less time you will have for your own personal study. In parallel to coursework and reading literature pertinent to your subdiscipline, take time to read about topics outside the scope of your current project, such as leading scientific trends in other fields and methods used in other communities that can be applied to your research. Research seminars presented in your home department and university are an excellent way to expose yourself to new ideas, network with technical experts from different areas, and enhance your general scientific and engineering literacy. Additionally, creating personalized alerts on Google Scholar, joining email lists of specific publishers and journals, and following researchers who are active on social media platforms (e.g., Linkedln, ResearchGate, Twitter), will allow you to stay current with the latest work.

As you approach the latter years of formal education and contemplate pursuing a career in academia, industry, or at a national laboratory, assess your current skills and values. Doing so will help identify the path that most interests you. There is an abundance of useful resources $^{1-5}$ that contrast the attributes, daily activities, and priorities between academic and industrial research. If your ambition is to become a faculty member, then obtaining a postdoctoral position that enables you to broaden your research base and blend a unique set of complementary investigative skills into a distinct vision for your independent research career will enhance your marketability. The reality is that there are relatively few tenure-track chemical engineering faculty openings compared to the number of applicants, so the competition for available positions is high. Diligent preparation and proper timing can increase your likelihood of success. One of the most critical tasks during this initial phase is to demonstrate your ability to publish high quality research in reputable, peer-reviewed journals pertinent to your area of specialty. The lack of a strong publication track record can prevent an otherwise promising candidate from advancing in the hiring process. This will also help inform your decision of whether or not to apply as a postdoc or during the final year of Ph.D. study; your publication track record should be commensurate with the expectations of someone in your field with your level of experience. For an additional perspective on the path to becoming an academic, we recommend reading The Professor Is In: The Essential Guide to Turning Your Ph.D. Into a Job by Dr. Karen Kelsky. ${ }^{6}$ Although it is written from the perspective of a professor in the humanities, this book contains valuable and practical information regarding the academic job search process and the advice is generally applicable to faculty candidates pursuing tenure-track positions across a range of disciplines.

Also, while in this preparation phase, there are several nontechnical skills you can build that will help you throughout your academic career. First and foremost, you must learn how to manage your time. Over the course of your career, your time is your most important commodity. If you begin to learn how to manage your time and how to integrate your work-life commitments, you will be more successful, healthier, and happier throughout your career. Second, a tip that has been used in the Davis lab is to keep a monthly record of your research expenses so that you are aware of the real operational costs of running a laboratory. This will assist in negotiating a faculty position and developing a meaningful request for startup funds. At Caltech, monthly expense reports are submitted by each researcher to Prof. Davis, and over the years, students have had fun testing whether 
or not he was reading these reports. For example, one student submitted a $\$ 450,000$ expense for a Ferrari! While this procedure does require the student to invest a bit of time away from scholarly pursuits, the reward is a better understanding of research costs that has been a great benefit to those launching independent careers.

\subsection{Develop communication skills early}

Now, more than ever, it is important to develop and hone your communication skills. In addition to becoming proficient in written (e.g., publications, proposals) and oral (e.g., presentations, lecturing) communications, you should also have a professional digital presence. The lack of a digital presence is now viewed as unusual. Mastery of all three elements of communication is important and essential to career success. Early on, you must learn to prioritize and implement nontechnical skills that complement technical knowledge and expertise. The ability to communicate with academics and nonacademics will be necessary throughout all career stages.

\subsection{Networking within the community}

Establishing and maintaining an active presence in your professional community is an important part of any discipline. Poster presentations and oral talks at local, regional, and national scientific meetings are prime opportunities to showcase your depth of fundamental knowledge to others in the field; however, sufficient preparation and practice are needed. You never want to give a bad talk in a public setting, nor engage in unfulfilling or inadequate discussions with audience members in the subsequent question and answer period. With the increased use of social media and "live-tweeting" at conferences, know that your presentations have the potential to reach secondary online audiences. Each presentation should be approached with the same level of care as a job interview, as first impressions can be difficult, if not impossible to overcome. You never know who will be in the audience or following your social media activity: it may be a future employer, colleague or collaborator, a future trainee or student in your lab, a future peer reviewer of one of your research manuscripts or proposals, a representative from a funding agency, or all of the above.

The conference forum should also be used to broaden your connections and cultivate relationships by meeting faculty, industrial professionals, and funding program managers. Having some familiarity with their work and being able to ask intelligent questions when introducing yourself to these individuals will help solidify a positive impression. Ultimately, some of the contacts you make may end up serving as referees by writing letters or reaching out to their network of colleagues at other universities to recommend you for opportunities later in your career. Thus, it is important to come prepared with business cards, especially for meetings with international participants. Additionally, it is important to follow up with people who you have met at conferences and meetings. A quick email saying that you enjoyed meeting the person is a great way to open the door to future interactions or collaborations.

\section{4 $\quad$ Utilizing social media and managing your online presence}

Building an effective, professional online presence to increase visibility is a prerequisite for emerging researchers who do not already have the benefit of being established, well-known independent investigators. Aside from the personal background and social media checks performed during employment screening, institutions increasingly rely upon searches of various online platforms to assess a candidate's professional reputation and research impact. While it is advisable to avoid negative representation online, a lack of internet presence is also a concern because most, if not all, people have some digital footprint. Use social media to your advantage as a researcher by taking control of your online presence in a strategic way that supports scholarship, teaching, and professional activities. ${ }^{7,8}$

The appropriate mix of tools and frequency of usage is left to the discretion of the individual user, but it is worthwhile to determine how others in your field use social media. Platforms such as Google Scholar, Linkedln, ResearchGate, and Twitter are widely used for chemical engineering and chemistry-related communication, while other fields such as mathematics and computational biology frequently use blogs to share research updates or discuss important issues in the discipline. These social networking services enable prospective faculty candidates to connect with other researchers in the field, disseminate their scholarly work, and substantiate their individual core competencies. Additionally, a personal website creates an avenue to tailor your individual brand in a consistent format that can be used to highlight your research interests and achievements, as well as provide centralized links to publications and other sites.

\section{I PHASE II: APPLYING TO FACULTY POSITIONS (6-9 MONTHS)}

Tenure-track faculty position listings typically begin to appear both online and in print during the late summer months and continue to emerge throughout the fall. While specific elements of the online application vary by institution and nature of the position, the typical package consists of a cover letter, curriculum vitae (CV), research and teaching statements, a list of reference letter writers, and a diversity and inclusion statement. Early preparation is crucial to allow sufficient time for thinking, reflecting, editing, and polishing your materials. Begin working on these documents in the late spring or early summer before the hiring cycle that you wish to apply. Additionally, familiarize yourself with the institutions and positions that you are applying to, and save a copy of each job posting for reference, because significant effort is required to tailor your application to each position. For example, doctoral universities with high to very high research activity (R1 and R2 universities by the Carnegie Classification of Institutions of Higher Education ${ }^{9}$ ) focus most heavily on the research statement and require that you clearly articulate a vision for launching an independent research program. Other schools, such as master's colleges and undergraduate institutions expect a greater focus on your teaching statement and demonstration of instructional abilities. 
Ideally, applications should be submitted several weeks before the AIChE Annual Meeting to allow search committees adequate time to review the materials and decide which applicants to visit during the Meet the Faculty Candidates poster session, and potentially arrange first-round interviews in-person at the AIChE meeting or by phone or video conferences shortly thereafter.

\section{1 $\quad$ Cover letter}

Each cover letter should be uniquely addressed to the university, department, and target position and concisely (1-2 pages) highlight your background, clearly state your research vision and interest in the position, and articulate a mutually beneficial fit between you and the department. Also, it is helpful to list the title, location, and time of your faculty candidate poster and any talks you will give at the AIChE Annual Meeting, and invite the search committee members to attend. This additional information can initiate requests for one-on-one meetings at the conference, and increase foot traffic at your faculty poster. Due to such customization of the cover letter, the tenure-track position search must be targeted (not generic), so careful thought must be given when deciding how many job openings to consider applying for.

A useful way to target your search is to begin assessing your potential fit at this stage within the department and university for each institution. Our advice is that you should only apply to a position if you can envision yourself accepting an employment offer from that school if one were to be extended. Job postings often include desired areas of specialty to be considered for the opening, and a careful review of the department website will allow you to evaluate how your skills align with existing expertise among the current faculty. Other factors to consider when assessing fit may include requirements for tenure and promotion, potential for collaboration, access to shared user facilities, campus culture and environment, and personal preferences such as geography, cost of living, climate, and so on.

\subsection{Curriculum vitae (CV)}

Your CV should be in a clear and concise format that includes only relevant information such as education, employment history, awards (specify national, local, or university level), publications, conference presentations, and any university and external service (e.g., manuscript reviewing, session chairing). If you obtained research funding as either a principal investigator (PI) or co-PI, then specify it accordingly, but do not include funding sources where you are not listed as a PI. Additional items to avoid include personal information, bullet points that are generic or cannot be substantiated with evidence, skills, or long descriptions of research activities. It is advisable not to list any papers in preparation, and ones that have been submitted to a target journal and are under review should be clearly marked as a separate list from peer-reviewed published work. Professional updates can be included on a personal website or blog that is easily accessible and searchable. For an excellent overview on how to prepare an academic CV, visit the University of California Berkeley's Career Center website. ${ }^{10}$

\section{3 $\quad$ Research statement}

The research statement is one of the most critical pieces of the faculty application package and should provide an overview of the type of research your program will conduct. It should emphasize the opportunities and challenges of your research area and field at a high level, while providing a few concrete examples of specific applications and research projects you will pursue in the first several years of your faculty career. Your research vision should be able to articulate how successful completion of these projects can contribute to establishing your research program and help contribute to meeting the grand challenges in your research field.

The research statement should begin with a summary (1 page or less) of the overall vision for your laboratory including how your research will be recognized and the types of problems that you will investigate. This summary should be understandable to any member of the department, not just the faculty experts in your particular subdiscipline. You want to captivate the entire department with this summary (once again, a key issue is knowing your audience). If you do not, it is unlikely that your application will be successful. Departments seek candidates who can establish programs with a core set of research skills and capabilities that not only address the important societal problems of today, but also can adapt to solve the unforeseen problems of tomorrow, over a decades-long career. As a faculty candidate, you want to carve out a space where your skills apply, but the ground is not already trampled. Thus, it is imperative to articulate a compelling research theme that differentiates you from your advisors and peers to avoid being the archetypal investigator. In some cases, students may have opened new areas of investigation and their advisors allow them to proceed with this area rather than the advisor (has occurred several times from the Davis lab at Caltech). If this is the case, then make this point clear in your research statement. There is nothing wrong with proceeding this way as a portion of your starting research, you just need to make this clear. For additional guidance on launching an independent research program, we recommend reading a recent perspective written by Professor Chris Jones (Georgia Tech). ${ }^{11}$

The core of the research statement focuses on several examples of specific research topics (2-3 pages each) with figures and references that will form the basis of the first set of projects you will assign to your graduate students and the initial proposals you will write for extramural funding. These research ideas should be well planned, including consideration of the target funding agencies and programs, and at least one idea should be unique and distinctive enough to define your research group in the broader community. Thus, the impression created by your research statement should not be generic or one that a department search committee can easily confuse with the research groups of other established professors or emerging faculty candidates. 


\section{4 $\quad$ Reference letter writers}

A list of professional references (typically 3-5) is included as part of the application package. Request permission from potential letter writers before listing them as references. The most effective letter writers are those who are able to provide a strong and detailed letter of support on your behalf, and tend to be individuals such as your research advisors (Ph.D. and postdoc) and close collaborators or committee members who know you well and are very familiar with your work and abilities. Other potential letter writers may be faculty members in your department who know you well, and have had experience evaluating former students at your institution for future faculty positions, and can speak to your potential scholarship, teaching, and mentoring abilities.

\section{5 $\quad$ Teaching and diversity statements}

While permissible to describe preferred instructional methods in the teaching statement, it is critical to state both the core chemical engineering courses, with specific course numbers and titles from the department's course catalog, that you can teach as well as those that you prefer to teach. Additionally, provide examples of electives or a special topics course you could teach, what the content will cover, and why the course is mutually beneficial to the department and your research group.

Many universities also require a diversity and inclusion statement as part of the faculty application package. While it is understandable that you may feel a bit unprepared to address these issues at this stage of your career, use this opportunity to articulate your views on why diversity and inclusivity are essential components of conducting impactful research and education in a university setting. Describe your vision for enhancing diversity and equity through your teaching, mentoring, and service activities. The office of diversity and inclusivity at your home institution is a great resource to use when preparing this statement, as they can assist you to better understand how you can promote diversity and enhance equity through recruitment, mentorship, and educational activities. By doing so, you will also learn how your university is fostering a more diverse and inclusive culture.

\section{I PHASE III: INTERVIEWING AND NEGOTIATING (3-6 MONTHS)}

What are the three most important issues with interviewing and negotiating? (a) Prepare, (b) prepare, and (c) prepare! There is no substitute for devoting time and effort to prepare for all aspects of these processes.

\section{1 | Interviewing}

The Meet the Faculty Candidates poster session at the AIChE Annual Meeting typically marks the beginning of the interviewing phase.
Many search committees will only visit your poster if they have already received and prescreened your application to determine that you may be of interest to interview for their open position. The poster session provides representatives from these departments an opportunity to meet you in person, and put a face to a name. As with every interaction while you are applying for positions, these are de facto interview situations.

The faculty candidate poster is used to show an overview of the research topics you have explored in the past and plan to explore in the future, and how your ideas will be competitive for extramural funding from specific agencies. Highlighting key outcomes and skill sets from your graduate and postdoctoral work is acceptable, but the majority of the poster should focus on your future research. A wellrehearsed and polished 30-60 s pitch will enable you to quickly communicate a clear, concise, and consistent message to visitors about the most important aspects of your proposed research program. Additionally, a Quick Response (QR) code that is linked to your supplemental material, personal website, or contact information is an effective method for directly connecting people to your work. For additional recommendations on effective poster presentations, refer to strategies discussed by Driskill. ${ }^{12}$

Use the remainder of the AIChE Annual Meeting to deliver oral presentations of your work (which may be attended by faculty search committee members), attend department receptions, introduce yourself to individual faculty, and have conversations with search committee members to discuss details of the tenure-track position while communicating your interest and research breadth, as appropriate.

When invited to video conference and on-campus interviews, you want to present a single, consistent expression of yourself and your skill set. Speak to faculty as if you are a peer, not a student, since implicit impressions are being made on your potential as a future colleague and perhaps one that they may spend the rest of their careers working with. Remember that all of your interactions during the interview process (e.g., meals, seminars, individual meetings, and coffee breaks) can impact your ability to receive an offer. Your objective is to leave a positive impression on everyone you meet from beginning to end, while simultaneously appearing confident (but not arrogant), relaxed, enthusiastic, engaging, and interested at all times. It is important to listen to questions in full and allow the speaker to completely finish before you answer. While others may interrupt your response, it is best to not interrupt the person engaging you in any interviewing situation.

The research seminar and the summary of original research and teaching plans, colloquially referred to as the "chalk talk," are the two most important aspects of the on-campus interview, and each require substantial preparation. Our advice is to focus the research seminar on one topic and go into as much fundamental detail as time will allow, because you will want to showcase your depth of knowledge. Many candidates try to split their presentation in half to talk about two separate topics: one from their Ph.D. thesis, and the other from their postdoc. Unless both topics can be logically integrated into a seamless lecture, this approach can be a mistake because it may fragment the seminar, leaving the audience confused and the message 
lost, and does not allow the opportunity to explain either topic in great depth. It is oftentimes more productive to pick one story and elaborate on it as much as possible. Although you want to showcase both depth and breadth, this should not come at the expense of depth. Begin the seminar with sufficient background information so that the entire audience can understand what the problem is and why it is important, keeping all engaged.

The chalk talk can vary from informal to formal, depending on the department, so clarify the preferred format with the search committee in advance. In general, the chalk talk should be kept at a high level and focus on your future research plans as opposed to reiterating material presented during the research seminar, weaving in common threads between proposed projects and highlighting the potential to address key fundamental questions in the field, while sustaining your laboratory for the first 5-10 years of your faculty career. Be prepared for a wide variety of questions from the faculty, many of which may be nontechnical, such as: "How will you differentiate yourself from your advisors?," or "What will you win an early career award for?" Be prepared to be interrupted during your chalk talk and do not allow it to throw you off track. Stay focused on answering questions and making your points while proactively managing your time so that all aspects of the talk are sufficiently addressed. For example, stage the sequence of proposed research topics in a logical fashion to facilitate discussion. If you do not know an answer to a question, say so. It is better to say, "I don't know, but I might expect $X$ based on $Y$ and $Z$," than to give a bogus response. No one knows the answers to everything, even though some faculty will try to give you the impression they do!

To prepare for these seminars, practice repeatedly with a live audience that may include peers, colleagues, or lab mates who can provide actionable feedback and recommendations on how to improve both the technical content and delivery of your talk.

\section{2 | Negotiating}

The standard startup package for a tenure-track assistant professor will typically consist of some or all of the following:

- Academic year salary, with summer salary support for a number of months

- Graduate student support in student-year equivalents

- Funds for capital equipment, consumable materials, and supplies

- Undergraduate research funds

- Discretionary funds for professional activities (e.g., memberships, travel, and entertainment)

- Anticipated teaching responsibilities and release policies

At each department, inquire about the flexibility and restrictions associated with funding. Funding for your startup package will likely originate from various sources (department, college, university), and be distributed differently at each university. This can place restrictions on the flexibility of how these initial funds can be used. For example, some departments will allow you to use graduate student support to instead hire a postdoctoral researcher (at the appropriate exchange rate), and some may provide one joint account to be used for all purchases (equipment, consumables, discretionary) while others will provide separate accounts for different types of purchases.

Ask for all of the offer terms in writing and review them with a trusted resource who is familiar with tenure-track job offers and negotiations. Before undertaking any negotiation, particularly with respect to equipment, materials, and supplies, first create two lists:

1. A wish list with all of the terms that you would ideally like, and;

2. A must-have list with the bare minimum terms that you can accept to startup your research program, as described in your research statement.

This exercise forces you to identify what is absolutely needed so that you can justify it to the department chair. You are responsible for maintaining any major equipment or instrumentation that you purchase, so consider whether it is something that will be utilized fulltime in your laboratory, or if you could manage by sharing time on a comparable instrument in another lab or user facility. Provide supporting justification, if needed, with budgetary quotes on larger instruments, and cost estimates on smaller pieces of equipment, lab supplies, tools, and so on. Request information on any training fees and hourly rates associated with the use of instruments from outside labs and access to shops and services. Additionally, reach out to new faculty members who have recently gone through this process and ask them for assistance. If your laboratory space requires renovations or additional infrastructure, first inquire if such costs will be charged against your start-up budget. Then, communicate the terms of these items in writing so that estimates can be obtained and the university can (attempt to) complete them before you start.

We recommend that you request salary support for the first two summers, but be prepared to receive less. Additionally, we recommend that you request some flexibility on the timing of your teaching release, and that the courses that you begin teaching in the first year also be assigned to you in future semesters, so that you do not need to teach new courses every semester. If given the opportunity to develop a new elective, then consider a course that can integrate teaching with some aspects of your research agenda. The required teaching commitments for research-focused tenure-track chemical engineering faculty will vary among institutions, but having some consistency in the courses you prepare and teach during the time period while you are establishing your research program (Phase IV) can be very helpful.

Finally, remember that the startup package is the one opportunity you have to negotiate the terms of your hire. Ask for what you need to be successful and identify what aspects you are willing to negotiate or forego. While you might not get everything you want, strategic negotiation can help you prioritize your greatest needs. 


\section{5 | PHASE IV: ESTABLISHING YOUR RESEARCH PROGRAM (5-6 YEARS)}

\section{1 | Building and managing a research group}

As a new $\mathrm{PI}$, you are responsible for setting the expectations in your laboratory from the start and creating a culture of scholarship, safety, professionalism, and ethics. Most importantly, you must be the intellectual leader in both your research (there is no substitute for a good idea) and mentorship. Your students will be influenced by how you handle difficult situations that inevitably arise from the challenging nature of conducting research and managing a diverse group of people, so your response is key to success. You need to select the "right" research questions to address and the "right" people (students, collaborators, and funders) to address them. Your first graduate students are critical in this regard, but they will look to you for training, guidance, and emphasis on what is most important. To facilitate their development, you need to be in the lab performing experiments and analyzing data alongside the students to ensure strict quality control. While tempting to get things up and running as quickly as possible to generate results, setting up the lab and training the first batch of students carefully and correctly from the onset will save time and frustration down the road.

Gradually, you need to phase out of the lab since there are many tasks, such as proposal and manuscript writing, that need to be done correctly, which no one else in the lab can do. Your time is limited: prioritize the critical tasks that only you can do and learn to say "NO" to the others. One of us (MED) is known to say, "What are the three most important points to starting a new faculty career?: (1) Learn to say no, (2) Learn to say no, and (3) Just say no!" While new faculty are often afraid to say "NO," it is critical that you begin your career with proper time management and avoid getting caught up with extraneous distractions at this stage. Focus on what you can control: mentoring students and performing excellent research that leads to publications with significance. Determine the work style, culture, and schedule that best suits you based on your strengths and weaknesses, and then build your research group around it accordingly. ${ }^{13}$ Multitasking is a skill that needs to be developed since you will be performing many functions including research, teaching, and mentoring. Some helpful strategies in this regard are to use the materials you develop more than once and to integrate teaching and research activities when possible. For example, review sections for research proposals can potentially be integrated into class materials or a review paper. Additionally, a selected topics elective that aligns with your research area and that of others in the department or university can be designed to help train graduate students and stay on the cutting edge of research developments. Teaching requires a tremendous investment of your time for course preparation, but the ability to teach the same courses more than once can lessen this effort in future offerings and enable you to demonstrate improvement in your instruction. For additional advice and helpful suggestions on teaching for tenure-track faculty, we recommend reading Advice for New Faculty Members: Nihil Nimus by Dr. Robert Boice. ${ }^{14}$
Recruit at least one (or more, if possible) highly-motivated, dependable, and productive graduate student from the first one to two incoming classes. These founding members will help generate the preliminary data for your first set of independent funding proposals and publications. They will set the tone of your laboratory and establish a culture that will last for years; thus, you should be selective with who you admit into your group. Taking no graduate student at all is better than taking a bad graduate student. A poor student will set your program back, which you cannot afford in time or money. Be cautious when recruiting graduate students and try to understand their motivation by determining why the student wants to join your lab as opposed to an established one, and why the student is pursuing a $\mathrm{Ph}$. D. in your research area. If given the opportunity to recruit students before you arrive on campus, conducting video conference interviews to prescreen them and obtaining recommendation letters will help in your assessment. If you are unsure, then refrain from recruiting the student until you arrive on campus and interact face-to-face.

Investing your efforts in developing graduate students for your lab can be a more fruitful long-term approach than hiring postdoctoral researchers at this early stage. It is possible to hire well-qualified, highcontributing postdocs as an assistant professor, but the best candidates are typically recruited by senior researchers. Unless a postdoc is personally recommended by someone you trust, there is potential for greater risk than reward. For additional guidance on the various aspects of creating and managing a research lab, we recommend reading Making the Right Moves: A Practical Guide to Scientific Management for Postdocs and New Faculty, Second Edition ${ }^{15}$ and other career tools available through the Burroughs Wellcome Fund website. ${ }^{16}$ While targeted toward academics in the biological and medical fields, the information in these resources are generally applicable to those initiating academic careers.

\section{2 | Developing your core research focus}

Now is the time to begin developing and refining your research goals for the next 5-10 years, which is a formidable undertaking when considering that the final results should have high impact, visibility and measurably move your research field forward. Working on significant problems requires a tremendous amount of time, even before you can begin to formulate the proper questions. ${ }^{17}$ The advantage of having such lofty goals, which most will recognize as worthy of pursuit, is that they lay a path for the incremental research advances leading up to them. Thus, when writing your first set of proposals, you can articulate and justify to reviewers how these initial small steps are required to contribute to achieving the loftier ultimate goal.

You should construct a research portfolio containing both highrisk, high-reward (i.e., home run) projects and low-risk, low-reward (i.e., singles, doubles) projects for the laboratory that will distinguish your program, as colorfully illustrated by the Ted Williams, hall of fame hitter-inspired, "heat map"18 in Figure 2. Not every home run swing will come to fruition, but you need to take some chances or you will likely not hit any. Do not underestimate the importance of lowrisk projects with the so-called "low-hanging fruit" that is needed to 


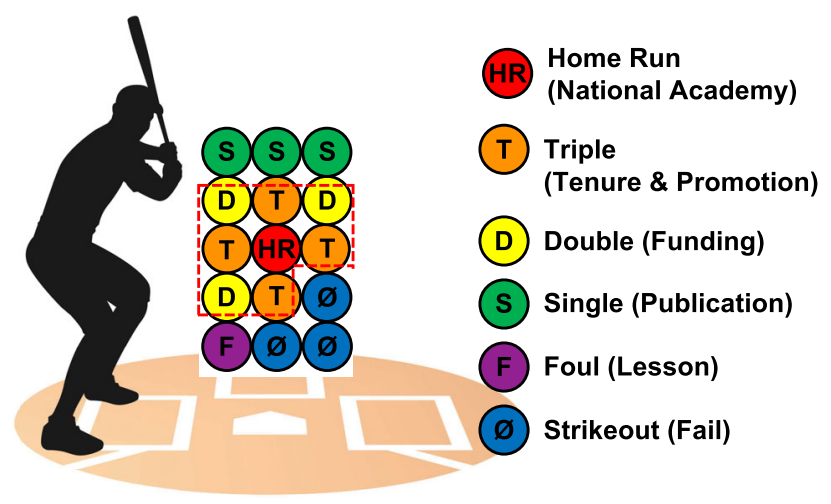

FIGURE 2 "Heat map" to illustrate new faculty research portfolio development strategy. Image inspired by "heat map" of baseball strike zone from The Science of Hitting by Williams and Underwood ${ }^{18}$ [Color figure can be viewed at wileyonlinelibrary.com]

establish productivity, publish papers, present at conferences and gather preliminary data for funding proposals, as long as such projects align with your research program objectives. Often these low-risk projects can be used to develop new competencies that may be linked together in the future to aim for some home run projects (inside-thepark home runs count the same!). While risk taking is important, the problems you select and the projects you start must be well thought out and done carefully. The opportunity cost at this stage is quite high since the majority of your flexible startup resources will likely be committed to these initial ideas, thus tying up a vast of amount of energy, time, and money at the expense of others. A key point is that one should not be afraid of failure and make projects so low-risk that their success provides little advancement to the field. The balance of highto low-risk projects is always difficult, but failures are to be expected and can lead to significant understanding. The key is to not make the same mistake twice!

Naturally, a steady stream of extramural funding is necessary to conduct impactful scientific research. However, it is advisable to wait, within reason, until you have a good idea to write a competitive proposal rather than submit a large number of poorly constructed, lowprobability ideas to each funding call that remotely falls within your field of research. The latter approach will consume a large amount of precious time and may impede your productivity. An efficient strategy is to also write some collaborative proposals with senior investigators. Multi-PI proposals are more frequently awarded than single-PI grants by certain agencies and this early funding can help prolong your startup funds and sustain your group during lean times. Also, do not underestimate the power of obtaining small, $\$ 5-10 \mathrm{~K}$ internal or external awards to string your program along. Ultimately, you will need to obtain larger, single-PI grants, but any external funding is better than no funding at all. Additionally, the research generated from collaborative, multi-PI grants will broaden your perspective, prompt new ideas for your core research portfolio, and generate results that can be leveraged as preliminary data in your future proposals. In a parallel effort, begin cultivating relationships with the program managers in your respective funding areas and volunteer to serve on proposal review panels for them: it will be an eye-opening experience that will greatly improve your own proposal submissions.

As you work to establish your research program on the path to tenure, identify at least one good mentor in your home department and one in your field from your network to consult with regarding how to navigate the academic environment and broader scientific community. Ask these mentors and respected colleagues to review your early grant proposals several weeks (or months) before submission. They will likely help you identify some fatal, yet correctible flaws, potentially saving you time and frustration with rounds of wasted peer-reviews. Ask for help and advice along the way, and find strategies to accelerate the learning curve.

\section{SUMMARY}

The path to becoming a tenure-track professor in chemical engineering is a rewarding but strenuous journey that is often initiated years earlier during one's Ph.D. studies. As a faculty candidate, obtaining pragmatic and actionable advice can be challenging considering the

TAB LE 1 Ten key guidelines for initiating a research-focused academic career in chemical engineering

\begin{tabular}{|c|c|}
\hline No. & Guideline \\
\hline I. & $\begin{array}{l}\text { Never give a bad talk; the same goes for your ability to answer } \\
\text { questions. Approach each talk as a job interview with the } \\
\text { audience. }\end{array}$ \\
\hline II. & $\begin{array}{l}\text { Cultivate relationships with members of your community, } \\
\text { from academia, national laboratories, and industry. They will } \\
\text { likely review your future manuscripts and research } \\
\text { proposals and potentially recommend you for professional } \\
\text { opportunities. }\end{array}$ \\
\hline III. & $\begin{array}{l}\text { Take control of your online presence, both personally and } \\
\text { professionally. Use social media and online platforms to } \\
\text { increase your visibility and impact. }\end{array}$ \\
\hline IV. & $\begin{array}{l}\text { Be excited about what you have to say. If you aren't excited, } \\
\text { then no one else will be. }\end{array}$ \\
\hline V. & $\begin{array}{l}\text { Develop a compelling research theme that distinguishes your } \\
\text { laboratory from your advisors and peers; avoid the } \\
\text { appearance of a "look-alike" program. }\end{array}$ \\
\hline VI. & $\begin{array}{l}\text { Be selective with your first group of graduate students. Taking } \\
\text { no student is better than taking a bad student. }\end{array}$ \\
\hline VII. & $\begin{array}{l}\text { Learn to say "NO." Manage and protect your time and mental } \\
\text { focus wisely by prioritizing your own research first. }\end{array}$ \\
\hline VIII. & $\begin{array}{l}\text { Identify a lofty research goal that will have high impact and } \\
\text { move the field forward, but lays a path for the lower-risk, } \\
\text { fruitful research that provides the stepping stones leading } \\
\text { to it. }\end{array}$ \\
\hline IX. & $\begin{array}{l}\text { Resist the temptation to apply for too many grants early on } \\
\text { until you have a good idea for how to craft a competitive } \\
\text { proposal. Submitting half-baked, low-probability ideas will } \\
\text { waste your time and ruin productivity. }\end{array}$ \\
\hline $\mathrm{X}$. & $\begin{array}{l}\text { Do not be afraid to take risks and fail; failure can be a great } \\
\text { teacher. Learn from your mistakes and use those lessons to } \\
\text { think more carefully in the future. The key is not to make } \\
\text { the same mistake twice. }\end{array}$ \\
\hline
\end{tabular}


abundance of information online and in print that is often contradictory and specific to other disciplines. To assist early career researchers, we have shared insights about processes related to faculty interviewing and hiring and initiating a research program in chemical engineering departments in the United States, which have collectively proven useful to new faculty members who formerly trained in the Davis lab at Caltech. The tenure-track career path was segmented into four phases along with approximate timelines, and the takeaway points from each of the four phases have been condensed into a summary of 10 key guidelines listed in Table 1.

In closing, we reiterate that this advice does not apply universally to all scientific disciplines, nor is it meant to be prescriptive. Rather, these perspectives are intended to assist prospective faculty candidates in navigating the interview and hiring process for researchfocused chemical engineering departments in the United States and launching his or her research program as effectively as possible while avoiding common pitfalls. Once you begin as a tenure-track faculty member, you will board a moving train that never comes to a stop. During these early years of your academic career, developing strategies to wisely manage and protect your time and mental focus, from an inundation of requests and demands on them, is paramount to success. Prioritize your research first, learn from your mistakes, and take luck when (and if) it comes, but do not count on it. ${ }^{15}$

\section{ACKNOWLEDGMENTS}

We are grateful to Prof. Christopher Paolucci (University of Virginia) and Prof. James W. Harris (University of Alabama) for helpful contributions by sharing their perspectives on pursuing a faculty position and starting a new research program. A special acknowledgment goes to Prof. W. Nicholas Delgass (Purdue University) for stimulating discussions and his implicit contributions to this perspective.

\section{REFERENCES}

1. Searls DB. Ten simple rules for choosing between industry and academia. PLoS Comput Biol. 2009;5(6):e1000388.

2. Bartholomew D. Academia or industry: where would I fit in? Science 2000; https://www.sciencemag.org/careers/2000/06/academia-orindustry-where-would-i-fit. Accessed October 24, 2019.

3. Bartholomew D. Academia or industry: finding the fit. Science. 2000; https://www.sciencemag.org/careers/2000/08/academia-orindustry-finding-fit. Accessed October 24, 2019.
4. Bartholomew D. Academia or industry: considering the fit. Science. 2000; https://www.sciencemag.org/careers/2000/08/academia-orindustry-considering-fit. Accessed October 24, 2019.

5. Maeda J. Academia vs. industry: the difference is in the punctuation Marks. Harv Bus Rev. 2009; https://hbr.org/2009/03/academia-vsindustry-the-diffe.html. Accessed October 24, 2019.

6. Kelsky K. The professor is in: the essential guide to turning your Ph.D. into a job. New York, NY: Three Rivers Press; 2015.

7. Mojarad S. Social media: more scientists needed. Science. 2017;357 (6358):1362-1363

8. Mojarad S. The future of chemistry communication is digital: overcoming hesitancies for online engagement. Communication in Chemistry. 2019;1327:243-262.

9. The Carnegie Classification of Institutions of Higher Education. Indiana University Center on Postsecondary Research. http:// carnegieclassifications.iu.edu. Accessed October 24, 2019.

10. Academic Job Search. University of California Berkeley Career Center. https://career.berkeley.edu/PhDs/PhDAcademic. Accessed October 24, 2019.

11. Jones CW. Advice for emerging researchers on research program development: a personal case study. AIChE J. 2017;63(9):3627-3635.

12. Driskill LP. Optimize your conference Poster's impact. Chem Eng Prog. 2010;106(8):43-48.

13. Wankat PC. The effective, efficient professor: teaching, scholarship, and service. 2nd ed. Boston, MA: Allyn and Bacon; 2002.

14. Boice R. Advice for new faculty members: Nihil Nimus. 1st ed. Boston, MA: Allyn and Bacon; 2000.

15. Guberman J, Saks J, Shapiro B, Torchia M. Making the Right Moves: A Practical Guide to Scientific Management for Postdocs and New Faculty. Howard Hughes Medical Institute and Burroughs Wellcome Fund 2006. https://www.hhmi.org/science-education/programs/makingright-moves. Accessed October 24, 2019.

16. Career Tools. Burroughs Wellcome Fund. https://www.bwfund.org/ career-tools. Accessed October 24, 2019.

17. Davis ME, Lobo RF. The "holey" grail: zeolites and molecular sieves. Chem Mater. 2018;30:5519-5520.

18. Williams T, Underwood J. The science of hitting. Revised ed. New York: Simon \& Schuster; 1986.

How to cite this article: Cybulskis VJ, Gounder R, Mojarad S, Davis ME. Initiating a research-focused academic career in chemical engineering: Perspectives from faculty at different career stages. AlChE J. 2020;e16927. https://doi.org/10. 1002/aic.16927 